\title{
Periodic Changes in Intensity and Arrival Time of Pulses from the Vela Pulsar: Evidence for Free Precession?
}

\author{
A.A. Deshpande \\ Raman Research Institute, Bangalore 560080, India \\ P.M. McCulloch \\ Physics Department, University of Tasmania, Box 252C, GPO Hobart, \\ Tasmania 7001, Australia
}

\section{Introduction}

We present dual-frequency measurements on the Vela pulsar with a view to study the slow variations in the pulsed flux and the apparent differences in the pulse arrival times. We examine the data for correlated variations between the pulse intensities and arrival times at the two frequencies and discuss two main possibilities in order to explain the observed behaviour.

\section{Dual-frequency measurements on the Vela pulsar}

The data presented here consists of a) Pulse intensities, $\mathrm{S}_{635} \& \mathrm{~S}_{950}$, at 635 \& $950 \mathrm{MHz}$ respectively and b) the 'residual' differences in the time of arrival of the pulse at the lower frequency, $\triangle \mathrm{TOA}$, with respect to that at the higher frequency. These data, over a span of $\sim 1300$ days (during 1988-92), were obtained as a part of the routine monitoring of the Vela pulsar from Mt. Pleasant Observatory of University of Tasmania, Hobart (see McCulloch et al. 1990).

The data are examined after block averaging over intervals of 5 sidereal days (to avoid any diurnal variations translating into annual variations) and are shown in Fig. 1. We have compared our data at $635 \mathrm{MHz}$ with those by Kaspi \& Stinebring (1992) at $610 \mathrm{MHz}$ over the common span and the agreement is excellent. The observed variations in $\mathrm{S}_{635} \& \mathrm{~S}_{950}$ correspond to a modulation index of about 10-15\% and show a high degree of correlation (60-70\%) between them. We also find that these intensity variations are anti-correlated with the relatively fast varying component of $\triangle \mathrm{TOA}$, with a correlation coefficient of -0.4 to -0.6 . The observed slow variation in $\triangle \mathrm{TOA}$ would be consistent with a reduction in the dispersion measure by about $0.025 \mathrm{pc} \mathrm{cm}^{-3}$.

Most importantly, when fourier analysed, the observed variations show strong periodicities corresponding to periods of $\sim 165 \pm 10$ days in $S_{635} \& S_{950}$ and of $\sim 330 \pm 20$ days in the $\triangle \mathrm{TOA} \& \mathrm{~S}_{635}$; quite distinct from annual variations.

\section{Refractive Scintillations or Free Precession ?}

While the observed correlations are consistent with those expected from refractive scintillations, the observed time-scales, particularly the periodicities, are 


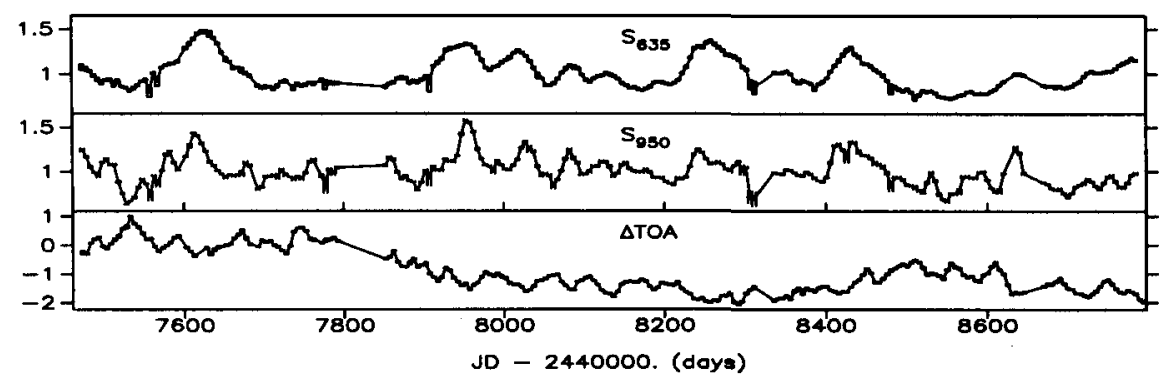

Figure 1. Normalised intensities of the Vela pulsar at $635 \& 950 \mathrm{MHz}$ and the TOA difference in milliperiods.

difficult to explain. Also, the observed modulation indices and the correlation between the intensities require the power-law index of the irregularity spectrum to be $>4.5$. Although this is not a strong indicator against refractive scintillations, it may be worth noting that if one corrects the intensity patterns, by removing the periodic modulation, the power-law index required is close to the Kolmogorov value.

Our data can be explained if we consider 'free precession' of the Vela pulsar with a wobble angle of a few degrees and a precession period of $\sim 330$ days. Cordes (1993) had already suggested such a possibility to explain changes in the intergrated pulse shape with a characteristic time-scales of about 100 days. The polarisation position angle sweep rates from available measurements range from -4 to $-9 \mathrm{deg} / \mathrm{deg}$ (Rankin 1993), a range consistent with a few degrees of wobble angle. Additional support for the possibility comes from the observation that the torque variations appear to dominate the timing noise over time scales of $\sim 300$ days and longer (D'Alessandro 1995).

While the case for 'refractive scintillations' can not be ruled out at present, the intersting possibility of 'free precession' seems more likely. If the Vela pulsar is indeed undergoing 'free precession' then we should look for a) correlated changes in the pulse shapes and the polarisation characteristics; b) whether the precession period has changed after the glitch in 1991 (JD >2448460).

If confirmed, this should provide valuable clues to better our understanding of the structure of neutron stars. Also, it will provide us a very useful means of probing the pulsar emission cone along the latitudinal direction.

\section{References}

Cordes, J. M. 1993, in Planets Around Pulsars (eds. Phillips, J.A., Thorsett, J.E. \&,Kulkarni, S.R.), pp. 43-60, Astro. Soc. of the Pacific, San Francisco.

D'Alessandro, F. 1995, Ph.D. Thesis, University of Tasmania.

Kaspi, V. M. \& Stinebring, D. R. 1992, ApJ, 392, 530.

McCulloch, P. M., Hamilton, P. A., McConnell, D. \& King, E. A. 1990, Nature, $346,822$.

Rankin, J. M. 1993, ApJ, 405, 285. 